\title{
Insetos nocivos a Prosopis sp. no Rio Grande do Norte (Brasil) e Piura (Peru).
}

RESUMO: A algarobeira (Prosopis sp.) é cultivada, no Brasil, principalmente na Região Nordeste, sendo que a sua introdução ocorreu a partir de 1942, em Serra Talhada, PE, com sementes procedentes de Piura, no Peru. Atualmente, é possível observar a presença de duas espécies no território brasileiro, a Prosopis juliflora e Prosopis pallida, as quais apresentam diversos benefícios para os agricultores locais. Esse levantamento foi feito com base em materiais encontrados e disponíveis na internet como: livros, artigos científicos, revistas e periódicos, no período de janeiro e fevereiro de 2019. Utilizaram-se as seguintes palavras chaves: Prosopis, Algaroba, Inseto, Rio Grande do Norte e Peru. Foram encontradas 16 espécies de insetos em associação daninha com Prosopis sp., no Rio Grande do Norte, distribuídas entre as ordens Coleoptera, Lepidoptera, Orthoptera e Hemiptera. Os registros de insetos em algaroba no Peru apresentaram maior abundância de dados e diversidades de ordens e famílias associadas à algaroba. No entanto, destacaram-se das listas encontradas, os insetos daninhos à planta, dos quais 18 espécies pertencem à Ordem Coleoptera e 11 espécies da Ordem Lepidoptera. Também foram registradas espécies das Ordens Hemiptera, Orthoptera, Thysanoptera e Diptera. No Brasil ocorrem espécies com potencial daninho à algaroba como, por exemplo, os do gênero Oncideres (Coleoptera: Cerambycidae), que poderiam ser mais bem estudados para o controle do Prosopis. As lagartas de Ascia momuste orseis (curuquerê-da-couve), conforme registros de ataque severo às folhas da algaroba, também merece atenção para o controle dessa espécie vegetal. O gênero Prosopis apresenta uma rica entomofauna no Peru, no entanto com poucos trabalhos de pesquisa no Brasil, o que não significa que não tenha uma entomofauna mais variada.

Palavras-chave: Algaroba, Dano, Entomofauna.

\section{Insects harmful to Prosopis sp. in Rio Grande do Norte (Brazil) and Piura (Peru)}

\begin{abstract}
The mesquite (Prosopis sp.) is cultivated in Brazil, mainly in the Northeast, and its introduction occurred in 1942, in Serra Talhada, PE, with seeds from Piura, Peru. Presently, it is possible to observe the presence of two species in the Brazilian territory, Prosopis juliflora and Prosopis pallida, which present several benefits for the local farmers. This survey was based on materials found and available on the Internet such as: books, scientific articles, journals and periodicals, in the period of january and february of 2019. The following keywords were used: Prosopis, Algaroba, Inseto, Rio Grande do North and Peru. Sixteen insect species were found in association with Prosopis sp., in Rio Grande do Norte county, distributed among Coleoptera, Lepidoptera, Orthoptera and Hemiptera Orders. The records of insects in mesquite in Peru presented greater abundance of data and diversities of orders and families associated to the mesquite. However, the insects harmful to the plant stand out from the lists found, of which 18 species belong to the Coleoptera Order and 11 species of the Lepidoptera Order. Species of the Orders Hemiptera, Orthoptera, Thysanoptera and Diptera were also registered. In Brazil there are species with harmful potential to the mesquite, such as those of the genus Oncideres (Coleoptera: Cerambycidae), which could be better studied for the control of Prosopis. The caterpillars of Ascia momuste orseis, according to records of severe attack on the leaves of the algaroba, also deserves attention for the control of this plant species. The genus Prosopis presents a rich insect variety in Peru, however with few research works in Brazil, which does not mean that it does not have a more varied insect fauna.
\end{abstract}

Key-words: Mesquite, Injuries, Insects.

\section{INTRODUÇÃO}

A algarobeira é uma árvore da família das leguminosas (Leguminoseae), pertencente ao gênero Prosopis, sendo conhecidas mais de 40 espécies, distribuídas em três continentes: América, Ásia e África, e encontra-se bem adaptada às condições climáticas do nordeste brasileiro, uma vez que pode atingir seu pleno desenvolvimento mesmo em solos com baixa fertilidade natural ou extremamente degradados (RIBASKI et al., 2009).

Segundo Ribaski et al. (2009), no Brasil é cultivada, principalmente, na Região Nordeste, sendo que a sua introdução ocorreu a partir de 1942, em Serra Talhada, PE, com sementes procedentes de Piura, no Peru. Existem também registros de duas outras introduções que foram realizadas em Angicos, $\mathrm{RN}$, em 1946, por meio de sementes oriundas do Peru e, em 1948, com sementes do Sudão.

Atualmente, é possível observar a presença de duas espécies no território brasileiro, a Prosopis juliflora e Prosopis pallida, as quais apresentam diversos benefícios para os agricultores locais, tais como madeira, resinas, corantes, fibras, sombreamento e disponibilização de recursos alimentares, como folhas e vagens, que são

\footnotetext{
Recebido em 05/07/2019, Aceito para publicação em 15/10/2019

${ }^{1}$ Universidade Federal Rural do Semi-Árido

*e-mail: diodato@ufersa.edu.br
} 
aproveitados por inúmeras espécies animais durante a estiagem (FRANCO et al., 2015a) (FRANCO et al., 2015b), período caracterizado principalmente pela ausência de chuvas e supressão da folhagem nas plantas caducifólias, típicas da caatinga (QUEIROZ et al., 2006).

A interação inseto-árvore, muitas vezes, nem sempre é benéfica para a planta e para o ser humano que a utiliza, ocorrendo, devido a isso, a preocupação de proteção da planta contra ataques maléficos de insetos-praga. Segundo Berti Filho (1979) os insetos da Ordem Coleoptera, por exemplo, destacam-se como os mais importantes dentre aqueles que são prejudiciais às essências florestais, não só pelo dano ocasionado como pela dificuldade de controle, dentre os quais destacam-se os coleópteros que são brocas e os vetores de doenças.

Por meio destes conhecimentos pode-se aplicar um manejo adequado para as essências florestais, a exemplo do controle de pragas, do combate a insetos daninhos, da escolha de espécies e locais para implantação das mesmas, entre outros.

A espécie em foco é considerada, no Brasil, como invasora e incentivado o seu desbaste como forma de combate à sua disseminação. Estudar a ocorrência de insetos inibidores da disseminação dessa essência florestal pode ser uma das medidas do seu controle. Sendo assim, o objetivo desse trabalho é o de verificar os registros da ocorrência de insetos prejudiciais à algaroba (Prosopis sp.), no Rio Grande do Norte, no Brasil, e no Peru.

\section{MATERIAIS E MÉTODOS}

Esse levantamento foi feito com base em materiais encontrados e disponíveis na internet como: livros, artigos científicos, revistas e periódicos, no período de Janeiro e fevereiro de 2019. Utilizaram-se as seguintes palavras chaves: Prosopis, Algaroba, Inseto, Rio Grande do Norte e Peru.

De todo o material encontrado foram utilizados artigos científicos, livro e comunicado técnico, a saber: Silva et al. (1997); Ramalho (1972); Santos (2015); Ribaski et al. (2009); Lima, Haji (1993) e Carvalho (1968).

No caso do Peru, foram analisados trabalhos de registros na localidade de Piura, já que é dessa região que houve a importação para o Brasil do gênero Prosopis. Listas exaustivas de insetos de ocorrência em algaroba encontraram-se nos trabalhos de Nuñez-Sacarias (1993) e Juárez et al. (2015).

\section{RESULTADOS E DISCUSSÃO}

Foram encontradas 16 espécies de insetos em associação daninha com Prosopis sp., no Rio Grande do Norte, distribuídas entre as ordens Coleoptera, Lepidoptera, Orthoptera e Hemiptera (Tabela 1). Na maioria dos casos a associação é com a família Heteroptera da ordem Hemiptera, e Cerambycidae, da ordem Coleoptera, que estão, respectivamente, associadas à algaroba atacando as folhas e as vagens.

Tabela 1. Gêneros e espécies de insetos e parte da algaroba (Prosopis sp.) injuriada, de acordo com levantamento realizado por diversos autores, no Rio Grande do Norte.

\begin{tabular}{|c|c|c|c|c|c|}
\hline Ordem/ Espécie & Família & $\begin{array}{l}\text { Parte da planta } \\
\text { injuriada }\end{array}$ & Ordem/ Espécie & Família & $\begin{array}{c}\text { Parte da } \\
\text { planta } \\
\text { injuriada }\end{array}$ \\
\hline Coleoptera & & & Orthoptera & & \\
\hline Nesozineus bucki & Cerambycidae & Vagem & Stiphra sp. & Proscopiidae & Folha \\
\hline Pandeleteius sp. & Curculionidae & Vagem & Schistocerca sp. & Acrididae & Folha \\
\hline Sitophilus sp. & Curculionidae & Vagem & Lepidoptera & & \\
\hline Lacioderma sp. & Ptinidae & Vagem & $\begin{array}{l}\text { Ascia momuste } \\
\text { orseis }\end{array}$ & Pieridae & Folha \\
\hline Mimosetes mimosae & $\begin{array}{c}\text { Chrysomelidae, } \\
\text { Bruchinae }\end{array}$ & Sementes & $\begin{array}{l}\text { Melipotis } \\
\text { ochrodes }\end{array}$ & Noctuidae & Folha \\
\hline Oncideres límpida & Cerambycidae & Ramos & Hemiptera & & \\
\hline Oncideres saga & Cerambycidae & Ramos & Dysdercus sp. & Pyrrochoridae & Folha \\
\hline Oncideres alicei & Cerambycidae & Ramos & Antitheucus sp. & Heteroptera & Folha \\
\hline $\begin{array}{l}\text { Retrachydes thoracicus } \\
\text { thoracicus }\end{array}$ & Cerambycidae & Ramos & Platycarenus sp. & Heteroptera & Folha \\
\hline
\end{tabular}

Em áreas de ocupação espontânea da algaroba Ribaski et al. (2009) descreveram que já foram encontradas grandes populações de agentes desfolhadores. Os mais agressivos são as lagartas Melipotis ochrodes (Guenée, 1852) e Ascia monuste orsei (Latreille). No estudo, os autores verificaram 
que as lagartas surgem no final da estação seca e, após o início das chuvas, diminuem.

As lagartas de Ascia momuste orseis (curuquerêda-couve) foram encontradas nos galhos e ramos das algarobeiras, devorando folhas novas e velhas. A intensidade do ataque deixou as árvores praticamente desfolhadas (LIMA, HAJI, 1993).

$\mathrm{O}$ gênero Stiphra, pertencente à Ordem Orthoptera, foi citado por Silva et al. (1997) e, provavelmente, trata-se da espécie Stiphra robusta Mello-Leitao, 1939 (nome comum: mané magro ou bicho pau), citada também por Ribaski et al. (2009), causando danos à algaroba de forma mais branda.

Também foram registrados, nos frutos das árvores, danos causados por besouros como o Lasioderma sp Stephens, 1835, no corpo da vagem, e nas sementes devido ao ataque do Mimosetes mimosae F. (RIBASKI et al., 2009).

Em Pernambuco, Carvalho et al. (1968) relataram que algumas algarobas existentes nos municípios de Gravatá, Bezerros e São Caetano, estavam sofrendo ataques de insetos serra-paus. $\mathrm{Na}$ região os pesquisadores identificaram ramos das árvores danificados por Oncideres límpida Bates. Em Soledade, na Paraíba, foram registrados ataques de Oncideres saga Dalman (RAMALHO, 1972). Ribaski et al. (2009) associaram como uma praga da espécie, outros serra-paus, como o Oncideres alicei Lane, 1977, Nesozineus bucki (Breuning, 1954) e Retrachydes thoracicus thoracicus (Olivier, 1790). Os autores identificaram que os insetos iniciam seus ataques aos ramos e galhos das algarobas a partir do segundo ano de idade da árvore.

Já, no caso do Peru (Tabela 2), foi possível observar o registro de uma maior abundância de dados e diversidades de ordens e famílias associadas à algaroba. No entanto, destacaram-se das listas encontradas, os insetos daninhos à planta, dos quais 18 espécies pertencem à Ordem Coleoptera, atacando raiz, tronco e vagem. Em segundo lugar os autores registraram 11 espécies da Ordem Lepidoptera, atacando folha e vagem da algaroba. As espécies das Ordens Hemiptera, Orthoptera, Thysanoptera e Diptera, destacaram-se pelo ataque apenas às folhas.

Conferiu-se que há poucas espécies em comum entre os registros realizados em ambos países. Em comum verificaram-se os gêneros Melipotis (Lepidoptera: Noctuidae), Dysdercus (Hemiptera: Pyrrhocoridae) e Schistocerca (Orthoptera: Acrididae).

A discrepância entre os registros em ambos países, provavelmente, deve-se às diferenças ambientais, tendo sempre em mente que essa espécie vegetal é exótica na região do nordeste brasileiro. Também pode ser razoável considerar que os estudos da fauna entomológicos de ocorrência na algaroba são poucos no Brasil, limitando-se mais a registrar os insetos que são mais evidentemente visualizados, devido, principalmente, a surtos de população.

Tabela 2. Insetos e parte da algaroba (Prosopis sp.) injuriada, de acordo com levantamento realizado por diversos autores, no Peru.

\begin{tabular}{|c|c|c|c|c|c|}
\hline Ordem/ Espécie & Família & $\begin{array}{l}\text { Parte } \\
\text { injuriada }\end{array}$ & Ordem/ Espécie & Família & $\begin{array}{c}\text { Parte da } \\
\text { planta injuriada }\end{array}$ \\
\hline Coleoptera & \multicolumn{5}{|c|}{ Lepidoptera } \\
\hline $\begin{array}{l}\text { Paranomala } \\
\text { undulata }\end{array}$ & Scarabaeidae & Raiz & Cnaphalocrocis sp. & Crambidae & Folha \\
\hline Cyclocephala sp. & Scarabaeidae & Raiz & Magusa sp. & Noctuidae & Folha \\
\hline Tomarus maimon & Scarabaeidae & Raiz & Melipotis trujillenis & Noctuidae & Folha \\
\hline Chrysobothris sp. & Buprestidae & Tronco & $\begin{array}{l}\text { Leploles } \\
\text { trigemmatus }\end{array}$ & Lycaenidae & Folha \\
\hline Agrilaxia sp. & Buprestidae & Tronco & $\begin{array}{l}\text { Plodia } \\
\text { interpunctella }\end{array}$ & Tortricidae & Vagem \\
\hline Ptinus sp. & Ptinidae & Tronco & Cryptophlebia sp. & Tortricidae & Vagem \\
\hline Micrapate humeralis & Ptinidae & Tronco & Laspeyresia sp. & Tortricidae & Vagem \\
\hline Heterachthes sp. & Cerambycidae & Tronco & Hemiptera & & \\
\hline Eburia pilosa & Cerambycidae & Tronco & Dechacona sp. & Cicadellidae & Folha \\
\hline $\begin{array}{l}\text { Sinoxylon } \\
\text { unidentatum }\end{array}$ & Bostrichidae & Tronco & Hemiberlesia sp. & Diaspididae & Folha \\
\hline Amphicerus & Bostrichidae & Tronco & Heterropsylla sp. & Psyllidae & Folha \\
\hline Achryson lineolatum & Bostrichidae & Tronco & Dysdercus sp. & Pyrrhocoridae & Folha \\
\hline Diabrotica speciosa & Cerambycidae & Folha & Orthoptera & & \\
\hline
\end{tabular}




\begin{tabular}{|c|c|c|c|c|c|}
\hline Amblycerus piurae & Chrysomelidae & Vagem & Schistocerca sp. & Acrididae & Folha \\
\hline Acanthoscelides sp. & Chrysomelidae & Vagem & Lactista sp. & Acrididae & Folha \\
\hline $\begin{array}{l}\text { Callosobruchus } \\
\text { maculatus }\end{array}$ & Chrysomelidae & Vagem & Orphulina sp. & Acrididae & Folha \\
\hline $\begin{array}{l}\text { Lasioderma } \\
\text { serricorne }\end{array}$ & Chrysomelidae & Vagem & Thysanoptera & & \\
\hline Gymnetis sp. & Scarabaeidae & Vagem & Frankliniella sp. & Thripidae & Folha \\
\hline Lepidoptera & & & Diptera & & \\
\hline Hypercompe sp. & Erebidae & Folha & Agromyza sp. & Agromyzidae & Folha \\
\hline Spoladea recurvalis & Erebidae & Folha & Enallodiplosis sp. & Cecidomyiidae & Folha \\
\hline $\begin{array}{l}\text { Hymenia } \\
\text { perspectalis }\end{array}$ & Crambidae & Folha & $\begin{array}{l}\text { Enallodiplosis } \\
\text { discordis }\end{array}$ & Cecidomyiidae & Folha \\
\hline Omiodes sp. & Crambidae & Folha & & & \\
\hline
\end{tabular}

Enfatiza-se a ausência do gênero Oncideres (Coleoptera: Cerambycidae) nos registros peruanos. No entanto, no nordeste brasileiro, foram assinaladas três espécies (Oncideres límpida, Oncideres saga e Oncideres alicei). As espécies desse gênero são consideradas, no Brasil, como importantes pragas florestais, o que sugere que poderiam ser estudadas como promissoras para o controle da algaroba, já que é considerada invasora nesse país.

\section{CONCLUSÕES}

O gênero Prosopis apresenta uma rica entomofauna no Peru, no entanto com poucos trabalhos de pesquisa no Brasil, o que não significa que não tenha uma entomofauna mais variada.

No Brasil ocorrem espécies com potencial daninho à algaroba como, por exemplo, os do gênero Oncideres (Coleoptera: Cerambycidae), que poderiam ser melhor estudados para o controle do Prosopis. As lagartas de Ascia momuste orseis (curuquerê-da-couve), conforme registros de ataque severo às folhas da algaroba, também merece atenção para o controle dessa espécie vegetal.

\section{REFERÊNCIAS}

BERTI FILHO, E. Coleópteros de importância florestal: 1 - Scolytidae. IPEF, Piracicaba, n. 19, p. 39-43, 1979.

CARVALHO, M. B.; CARVALHO, E. P.; ARRUDA, G. P. de. O "Serrador" praga da algarobeira. Recife: Instituto de Pesquisas Agronômicas de Pernambuco, Boletim Técnico n. 33, 1968. 26p.

FRANCO, E. S.; DANTAS-NETO, J.; GUIMARÃES, J. P.; FARIAS, M. S. S.; LIRA, V. M. Comparação de indicadores químicos do solo após o plantio da algaroba. Agropecuária Científica do Semiárido, Patos, v. 11, n. 2, p. 61-66, abr./jun. 2015a.
FRANCO, E. S.; DANTAS-NETO, J.; GUIMARÃES, J. P.; FARIAS, M. S. S.; LIRA, V. M. Influência das diferentes lâminas de irrigação no crescimento da algaroba. Agropecuária Científica do Semiárido, Patos, v. 11, n. 2, 67-71, abr./jun. 2015 b.

JUÁREZ， G.; GRADOS， N.; CRUZ， G. Insectos asociados a Prosopis pallida (Humb. \& Bonpl. ex. Wild.) en el campus de la Universidad de Piura, Perú. Zonas Áridas, Lima, Peru, n. 16, pg. 1-24, 2016.

LIMA, P. C. F.; HAJI, F. N. P. Ocorrência de desfolhamento por lagartas em algarobeira no trópico semiárido do Brasil. Parte do Boletim de Pesquisa Florestal, Colombo, n.26/27, p.57-59, Jan./Dez. 1993.

NUÑEZ-SACARIAS, E. Insectos del algarrobo (Prosopis spp) en el Perú: Costa norte (Piura) Costa central (Ica). Revista Peruana de Entomología. n. 36, pg.69-83, 1993.

QUEIROZ, L. P.; RAPINI, A.; GIULIETTI, A. M. Rumo ao Amplo Conhecimento da Biodiversidade do semiárido brasileiro. Brasília: Centro de Gestão e Estudos Estratégicos, 2006. 144 p.

RAMALHO, F. S. Nota sobre o "Serrador" da (Coleoptera Cerambycidae) algarobeira. Oncideres saga (Dalman, 1823). Pesquisa Agropecuária do Nordeste, v.4, n.2, p.11-12, 1972.

RIBASKI, J.; DRUMOND, M. A.; OLIVEIRA, V. R.; NASCIMENTO, C. E. de S. Algaroba (Prosopis juliflora): Árvore de uso múltiplo para a região Semiárida Brasileira. Comunicado Técnico 240, Colombo, 2009.

SANTOS, J. P. S. dos. A algaroba no Rio Grande do Norte: histórico, usos e potencialidades dos recursos da árvore. Mossoró: Edição do autor, 2015, 133p.

SILVA, J. A. P. da; CHAVES, J. W. N.; FILGUEIRA, M. A; SANTOS, J. H. R. dos. Biocenose da algaroneira (Prosopis juliflora (sw.) dc.) com ênfase nos insetos fitófagos e seus predadores. Revista Caatinga, Mossoró, v. 10, n. $1 / 2,1997$. 